\title{
Perfil antropométrico, somatotipo y composición corporal de jóvenes jugadores de tenis de mesa. \\ Anthropometric profile, somatotype, and body composition of young table tennis players.
}

\author{
Pradas de la Fuente, Francisco \\ Facultad de Ciencias de la Salud y del Deporte. Universidad de Zaragoza \\ Carrasco Páez, Luís \\ Facultad de Ciencias de la Educación. Universidad de Sevilla \\ Martínez Pardo, Esmeraldo \\ IES Cabo de la Huerta. Alicante \\ Herrero Pagán, Rafael \\ Real Federación Española de Tenis de Mesa
}

\section{Resu m e n}

El objetivo de este trabajo es determinar las características cineantropométricas de jóvenes jugadores españoles de tenis de mesa de nivel nacional e internacional. Un total de sesenta y tres jugadores (treinta y ocho chicos y veinticinco chicas), fueron divididos en tres grupos atendiendo a sus edades (G1, hasta 11 años; G2, 11 y 12 años; G3, 13-14 años). Se registraron diferentes medidas corporales: peso, talla, pliegues cutáneos (bíceps, tríceps, subescapular, suprailiaco, supraespinal, abdominal, muslo anterior y pierna medial), perímetros (brazo relajado, brazo tensado y flexionado, muslo medio y pierna) y diámetros (biepicondíleo de húmero, biestiloideo de muñeca y bicondíleo de fémur). Además del correspondiente análisis antropométrico, se realizaron cálculos para determinar la composición corporal y el somatotipo de los sujetos participantes en el estudio. Así el somatotipo general del grupo resultó ser de tipo endomórfico-mesomórfico, coincidiendo con el de los grupos G2 y G3. El análisis teniendo en cuenta el factor género, puso de manifiesto un somatotipo mesomórfico balanceado en el grupo de varones, y meso-endomórfico en el de mujeres. Los datos correspondientes a la composición corporal y contrastados según el factor género mostraron un mayor porcentaje graso en mujeres respecto a varones y un porcentaje óseo más alto en el grupo de varones con respecto al de mujeres. Estos resultados coinciden, en parte, con los encontrados en otros estudios realizados con jugadores de tenis de mesa, en los que se ha observado, de forma global, un predominio equilibrado de los componentes mesomórfico y endomórfico sobre el componente ectomórfico.

\begin{abstract}
The aim of this investigation is to determine the anthropometric characteristics of Spanish young table tennis players with national and international competition level. Sixty-three players (thirty-eight males and twenty-five females), were divided in three age-groups (G1, until 11 years; G2: $11-12$ years and G3: $13-14$ years old). Different body measures were registered: body mass, height, skinfolds (biceps, triceps, subscapular, suprailiac, supraspinale, abdominal, anterior thigh, and medial calf), girths (arm flexed and relaxed; arm flexed and tensed, thigh, and calf) and breadths (biepicondylar of the humerus, bistyloid, and biepicondylar of the femur). In addition to anthropometric analysis, several calculations were performed to determine body composition and somatotype of participants. In this sense, a mesomorph - endomorph somatotype was registered for total group, coinciding with somatotype of $\mathrm{G} 2$ and $\mathrm{G} 3$ groups. Anlysis take into account the gender factor revealed a balanced mesomorph somatotype for males and a mesomorphic endomorph somatotype for females. Data corresponding to body composition and contrasted with gender show higher body fat percentage in females than in males and higher bone mass percentage in males than in males. This results are, in part, similar to those in other studies carried out with table tennis players in which it has been observed a prevalence of mesomorph and endomorph components over ectomorph one.
\end{abstract}

Key words: table tennis, anthropometrics, body composition, somatotype.

Palabras clave: tenis de mesa, antropometría, composición corporal, somatotipo.

Correspondencia/correspondence: Pradas de la Fuente, Francisco

Facultad de Ciencias de la Salud y del Deporte. Universidad de Zaragoza

E-mail: franprad@unizar.es 
Pradas de la Fuente, F; Carrasco Páez, L.; Martínez Pardo, E.; Herrero Pagán, R. (2007). Perfil antropométrico, somatotipo y composición corporal de jóvenes jugadores de tenis de mesa. Revista Internacional de Ciencias del Deporte. 7(3), 11-23 http://www.cafyd.com/REVISTA/00702.pdf

\section{Introducción}

$\mathrm{E}^{\mathrm{s}}$ tenis de mesa, es un deporte con características acíclicas, en el que se suceden continuamente, periodos de trabajo y de descanso. Los constantes cambios de ritmo e intensidad que se producen durante los partidos, lo convierten en una actividad mixta desde el punto de vista del metabolismo energético.

Entre las cualidades físicas más importantes de sus jugadores, cabe mencionar la velocidad y la resistencia, ya que los cortos esfuerzos de actividad intensa durante el juego, para los que se emplea fundamentalmente un metabolismo de tipo anaeróbico, precisan un entrenamiento de la velocidad, mientras que la capacidad de afrontar adecuadamente la duración de la competición, depende en cierta medida, de un trabajo de resistencia aeróbico (Bagur, 1996). No obstante, la fuerza, la coordinación y la flexibilidad, también desempeñan un papel crucial en este deporte (Bermejo, 1991).

Aunque resulta obvio que el entrenamiento físico juega un papel fundamental en la consecución del éxito deportivo, la realidad, es que no existen procedimientos capaces de modificar de manera significativa los límites impuestos por la naturaleza. Diversos estudios han demostrado que, a igualdad de condiciones de entrenamiento físico, tanto desde el punto de vista cuantitativo como cualitativo, los mejores resultados deportivos, corresponden a aquellos sujetos con unas condiciones anatómicas más favorecedoras para la práctica del deporte en cuestión, considerando las características antropométricas parte del conjunto de variables biológicas relacionadas con el rendimiento deportivo (Esparza, 1993).

En términos biotipológicos, esta relación entre estructura y función a la que se acaba de hacer referencia, también ha sido demostrada a partir de estudios observacionales, en los que se ha comprobado que atletas de una misma modalidad, con resultados deportivos similares, manifestaban tipos y formas corporales muy semejantes (Zatsiorski, 1989).

Por otra parte, el concepto de biotipo debe ser entendido de una forma dinámica, entrenable y modificable, únicamente, hasta el límite marcado por la carga genética individual. Este matiz, que parece restar valor al estudio de la condición anatómica como elemento de ayuda en el ámbito del rendimiento deportivo, no debe ser considerado en un sentido negativo, sino que debe servir para atribuirle el peso real que posee, evitando incurrir en el no infrecuente error de sobrevalorar su papel en el deporte, o por el contrario, subestimarlo.

Hoy día, el estudio de la forma humana constituye una herramienta de gran interés, tanto en la selección precoz de la modalidad deportiva más adecuada para un sujeto de acuerdo con sus cualidades anatómicas, como en el control de la eficacia de un programa de entrenamiento, sin olvidar que las posibles modificaciones experimentadas por estas variables en el transcurso del tiempo no suelen adquirir grandes magnitudes, siendo preciso entender la importancia que poseen las pequeñas variaciones en este contexto, como indicadores de control.

Muchos autores de gran prestigio en el campo del entrenamiento deportivo, como Bompa (1987), defienden la importancia de descubrir a una edad temprana a los individuos más capacitados para una especialidad deportiva, seleccionarlos, y llevar a cabo un proceso continuo de seguimiento, con el fin de facilitar la consecución del nivel más elevado de dominio de su deporte.

Por tanto, considerando por una parte la importancia de la detección precoz de talentos en el proceso formativo de deportistas de alto nivel y, por otra, la influencia del biotipo en el 
Pradas de la Fuente, F; Carrasco Páez, L.; Martínez Pardo, E.; Herrero Pagán, R. (2007). Perfil antropométrico, somatotipo y composición corporal de jóvenes jugadores de tenis de mesa. Revista Internacional de Ciencias del Deporte. 7(3), 11-23 http://www.cafyd.com/REVISTA/00702.pdf

rendimiento deportivo y su utilidad como medida objetiva de control del entrenamiento, sin olvidar el desconocimiento actual que aún existe sobre la condición física idónea de los jugadores en tenis de mesa, se puede deducir el interés de poner en marcha estudios que permitan desarrollar tablas antropométricas de referencia, específicas para este deporte, que faciliten tanto el proceso de detección de jóvenes con condiciones anatómicas altamente favorables para desarrollar sus habilidades deportivas, como la estandarización de la evaluación objetiva de estos jugadores, a lo largo del periodo formativo.

Con todo lo anterior, el principal objetivo del presente estudio es establecer el perfil antropométrico, el somatotipo, así como diferentes componentes relacionados con la composición corporal en jóvenes jugadores de tenis de mesa que faciliten el proceso de detección precoz de jóvenes con condiciones anatómicas favorables para desarrollar sus habilidades en esta disciplina deportiva y que puedan utilizarse, además, como medidas objetivas en el control del entrenamiento.

\section{Material y métodos}

Un total de 63 jugadores de tenis de mesa (38 varones y 25 mujeres; edad: $11.4 \pm 1.8$ años), seleccionados para el Programa Nacional de Tecnificación Deportiva de la Real Federación Española de Tenis de Mesa, participaron en el estudio. Atendiendo a la categoría a la que pertenecían los jugadores en el momento de realizar esta investigación, se conformaron tres grupos: G1, compuesto por 22 jugadores (15 chicos y 7 chicas) de categoría benjamín; G2, compuesto por 27 jugadores (15 chicos y 12 chicas) de categoría alevín y G3, compuesto por 14 jugadores (8 chicos y 6 chicas) de categoría infantil. En cualquier caso, estos sujetos entrenaban un mínimo de $8 \mathrm{~h}$ semanales, compitiendo en eventos de nivel nacional e internacional.

Las medidas antropométricas practicadas fueron: peso, talla, pliegues cutáneos (bíceps, tríceps, subescapular, suprailíaco, supraespinal, abdominal, muslo anterior y pierna medial), perímetros (brazo relajado, brazo contraído, muslo medio y pierna) y diámetros (biepicondíleo, biestiloideo y bicondíleo). Para el registro de estas medidas se aplicó el protocolo propuesto por la International Society for the Advancement of Anthropometry (ISAK), utilizando, para ello, material básico de antropometría (báscula y tallímetro modelo Seca 714, con precisión de 100 g y 1mm, respectivamente; lipómetro modelo Holtain, con precisión de $0.2 \mathrm{~mm}$; paquímetro modelo Holtain, con precisión de 1mm; cinta antropométrica Holtain, con precisión de 1mm y lápiz dermográfico).

A partir de las medidas antropométricas valoradas se calcularon, además, diferentes índices y porcentajes con el fin determinar la composición corporal de los jugadores participantes en el estudio. Así, se calculó el índice de masa corporal (IMC, expresado en $\mathrm{kg} / \mathrm{m}^{2}$ )

En cuanto a la composición corporal, se utilizaron los cálculos propuestos por De Rose y Guimaraes (1980), basados en un modelo tetracompartimental. Así, se determinaron los componentes graso, óseo, muscular y residual. Aunque las fórmulas aplicadas para determinar los distintos componentes corporales proporcionan los resultados numéricos en términos absolutos (en kilogramos), para poder efectuar comparaciones entre sujetos, todos los valores obtenidos, fueron corregidos, expresándose de forma porcentual en relación al peso corporal de cada sujeto. 
Pradas de la Fuente, F; Carrasco Páez, L.; Martínez Pardo, E.; Herrero Pagán, R. (2007). Perfil antropométrico, somatotipo y composición corporal de jóvenes jugadores de tenis de mesa. Revista Internacional de Ciencias del Deporte. 7(3), 11-23 http://www.cafyd.com/REVISTA/00702.pdf

El análisis del somatotipo se realizó mediante el método de Heath-Carter, obteniéndose el valor de los tres componentes: endomórfico, mesomórfico y ectomórfico (Carter, 1990). Asimismo, se calculó la distancia de dispersión de dos somatotipos medios para comparar los somatotipos de los grupos estudiados con somatotipos de referencia (Esparza, 1993).

Todos los datos obtenidos se muestran como media \pm desviación típica (dt). Se aplicó la prueba de Kolmogorov-Smirnov para así definir el tipo de distribución de cada una de las variables consideradas. Atendiendo a los resultados de esta prueba, y con la intención de establecer una comparativa entre los grupos establecidos, se aplicó metodología paramétrica (Prueba T de Student y análisis de la varianza) cuando las variables de contraste seguían una distribución normal y metodología no paramétrica (U de Mann Whitney y prueba de Kruskal Wallis) cuando estas variables no seguían este tipo de distribución. Todos estos cálculos se realizaron con el paquete estadístico SPSS v12.0 para Windows. En todo caso se estableció un intervalo de confianza del 95\%.

\section{Resultados}

En la Tabla 1 se muestran las características generales de los sujetos participantes en el estudio distribuidos en grupos según su categoría y género, observándose diferencias significativas entre los categorías benjamín, alevín e infantil para las variables peso, talla e índice de masa corporal.

\begin{tabular}{|c|c|c|c|c|c|c|}
\hline GRUPOS & GÉNERO & ESTADÍSTICO & $\begin{array}{l}\text { Edad } \\
\text { (años) }\end{array}$ & $\begin{array}{l}\text { Masa corporal } \\
(\mathrm{kg})\end{array}$ & $\begin{array}{l}\text { Talla } \\
(\mathrm{cm})\end{array}$ & $\begin{array}{c}\mathrm{IMC} \\
\left(\mathrm{kg} / \mathrm{m}^{2}\right)\end{array}$ \\
\hline \multirow{6}{*}{$\begin{array}{c}\mathrm{G1} \\
\text { (benjamín) }\end{array}$} & \multirow{2}{*}{$\begin{array}{l}\text { MASCULINO } \\
n=15\end{array}$} & Media & 9,67 & 35,88 & 142,09 & 17,71 \\
\hline & & Dt & ,49 & 6,57 & 6,91 & 2,36 \\
\hline & \multirow{2}{*}{$\begin{array}{c}\text { FEMENINO } \\
n=7\end{array}$} & Media & 9,57 & 34,18 & 140,10 & 17,24 \\
\hline & & $\mathrm{Dt}$ &, 53 & 7,94 & 5,27 & 2,98 \\
\hline & \multirow{2}{*}{$\begin{array}{l}\text { Total } \\
n=22\end{array}$} & Media & 9,64 & 35,3455 & 141,45 & 17,56 \\
\hline & & $\mathrm{Dt}$ & ,492 & 6,88966 & 6,38 & 2,51 \\
\hline \multirow{6}{*}{$\begin{array}{c}\text { G2 } \\
\text { (alevín) }\end{array}$} & \multirow{2}{*}{$\begin{array}{c}\text { MASCULINO } \\
n=15\end{array}$} & Media & 11,40 & 38,45 & 146,73 & 17,77 \\
\hline & & $\mathrm{Dt}$ &, 51 & 6,13 & 6,41 & 1,76 \\
\hline & \multirow{2}{*}{$\begin{array}{c}\text { FEMENINO } \\
n=12\end{array}$} & Media & 11,25 & 45,66 & 151,36 & 19,79 \\
\hline & & $\mathrm{Dt}$ & ,45 & 10,07 & 10,02 & 3,35 \\
\hline & \multirow{2}{*}{$\begin{array}{l}\text { Total } \\
\mathrm{n}=27\end{array}$} & Media & 11,33 & 41,6556 & 148,78 & 18,67 \\
\hline & & $\mathrm{Dt}$ & ,480 & 8,74578 & 8,37 & 2,73 \\
\hline \multirow{6}{*}{$\begin{array}{c}\mathrm{G} 3 \\
\text { (infantil) }\end{array}$} & \multirow{2}{*}{$\begin{array}{c}\text { MASCULINO } \\
n=8\end{array}$} & Media & 14,25 & 58,30 & 167,10 & 20,68 \\
\hline & & $\mathrm{Dt}$ & ,88 & 13,02 & 10,76 & 2,87 \\
\hline & \multirow{2}{*}{$\begin{array}{c}\text { FEMENINO } \\
n=6\end{array}$} & Media & 14,50 & 53,62 & 161,58 & 20,51 \\
\hline & & $\mathrm{Dt}$ & 1,05 & 7,24 & 3,79 & 2,50 \\
\hline & \multirow{2}{*}{$\begin{array}{l}\text { Total } \\
\mathrm{n}=14\end{array}$} & Media & $14,36^{* *}$ & $56,29^{* *}$ & $164,73^{\text {*t }}$ & $20,61^{\text {* }}$ \\
\hline & & $\mathrm{Dt}$ & ,93 & 10,83 & 8,71 & 2,62 \\
\hline \multirow{6}{*}{ TOTAL } & \multirow{2}{*}{$\begin{array}{c}\text { MASCULINO } \\
n=38\end{array}$} & Media & 11,32 & 41,61 & 149,18 & 18,36 \\
\hline & & $\mathrm{Dt}$ & 1,82 & 11,84 & 12,16 & 2,51 \\
\hline & \multirow{2}{*}{$\begin{array}{c}\text { FEMENINO } \\
n=25\end{array}$} & Media & 11,56 & 44,36 & 150,66 & 19,25 \\
\hline & & $\mathrm{Dt}$ & 1,94 & 11,21 & 10,89 & 3,22 \\
\hline & \multirow{2}{*}{$\begin{array}{c}\text { TOTAL } \\
\mathrm{n}=63\end{array}$} & Media & 11,41 & 42,70 & 149,77 & 18,71 \\
\hline & & Dt & 1,85 & 11,58 & 11,60 & 2,82 \\
\hline
\end{tabular}

Tabla 1. Características generales por grupos de categorías deportivas y de género IMC= Índice de Masa Corporal.

** Diferencias significativas $(\mathrm{p}<0,01)$ entre los grupos G3 y G1-G2. 
Pradas de la Fuente, F; Carrasco Páez, L.; Martínez Pardo, E.; Herrero Pagán, R. (2007). Perfil antropométrico, somatotipo y composición corporal de jóvenes jugadores de tenis de mesa. Revista Internacional de Ciencias del Deporte. 7(3), 11-23 http://www.cafyd.com/REVISTA/00702.pdf

El análisis por grupos teniendo en cuenta la variable género puso de manifiesto diferencias significativas en lo que hace referencia a los componentes óseo, residual y graso, siendo los dos primeros superiores en el género masculino, y el último en el femenino (Figura 1).

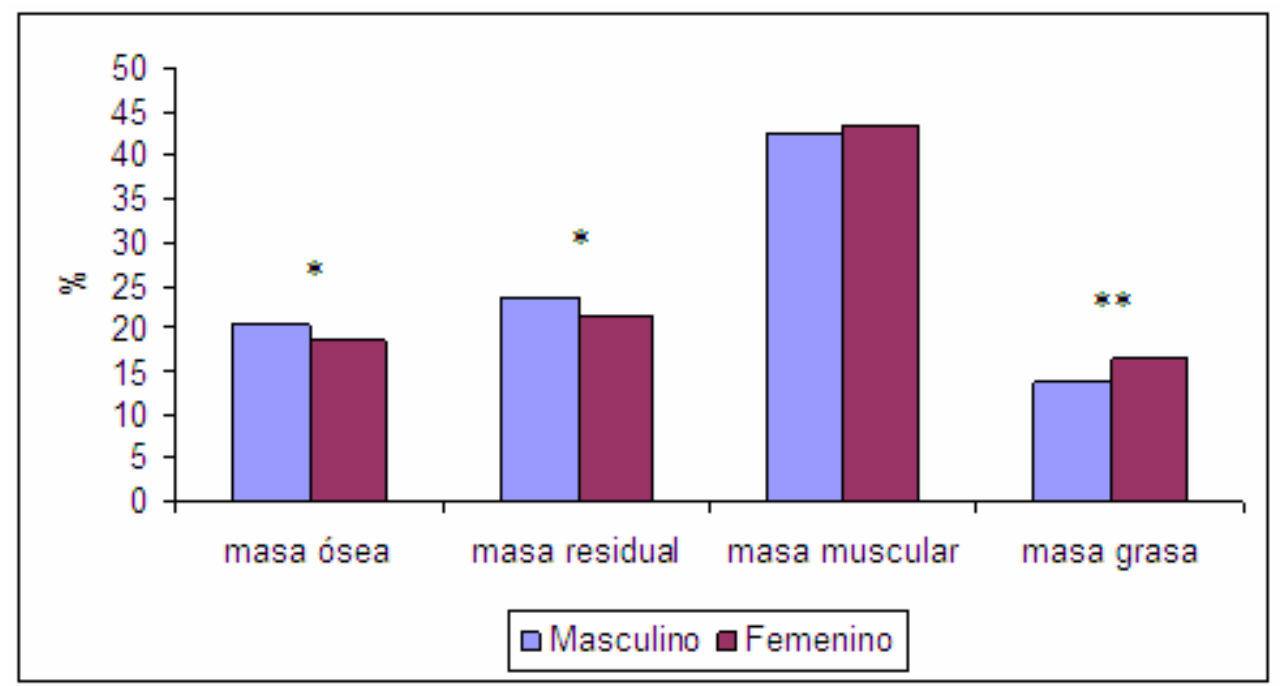

Figura 1. Composición corporal de los deportistas estudiados atendiendo a la variable género. ${ }^{*} \mathrm{p}<0.05 ;{ }^{* *} \mathrm{p}<0.01$.

En cuanto al análisis de la composición corporal por categorías, los valores más elevados en relación con el porcentaje óseo, fueron encontrados en el grupo G1 de género masculino, no existiendo diferencias significativas con los otros grupos y tampoco con las chicas del mismo grupo (Figura 2).

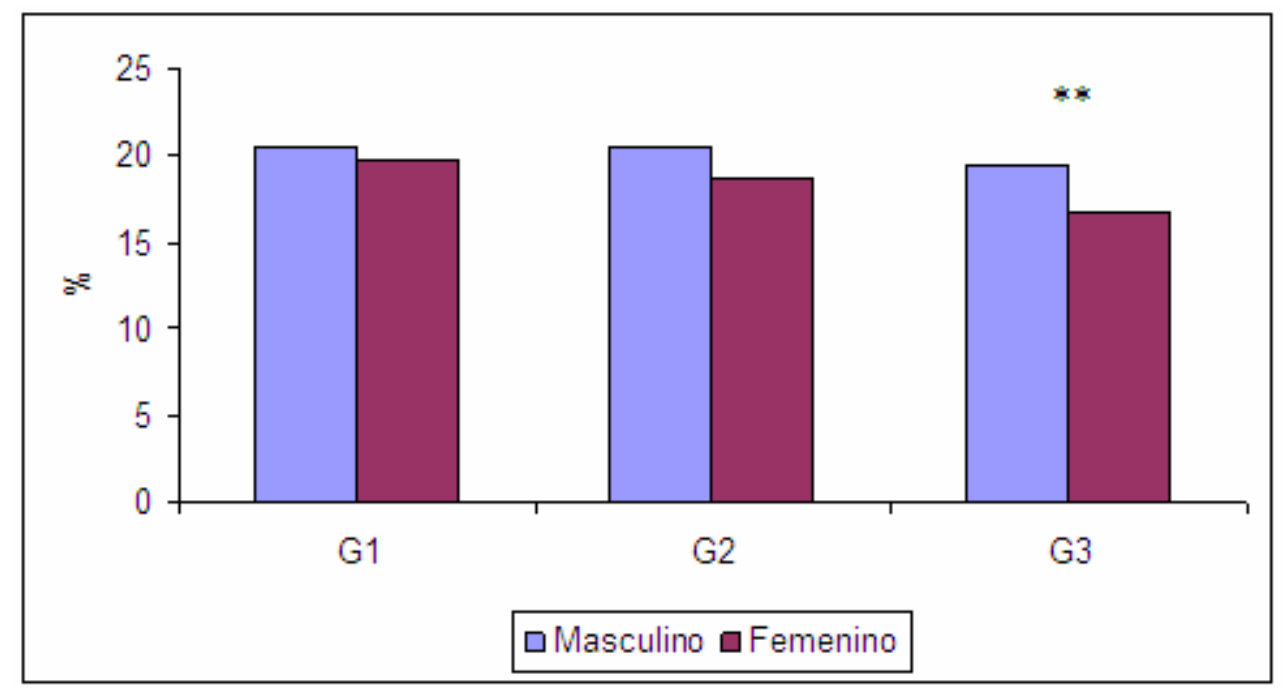

Figura 2. Componente óseo (porcentaje sobre la masa corporal) de los deportistas estudiados. ${ }^{* *} \mathrm{p}<0.01$.

Al evaluar el componente óseo por grupos de género dentro de cada categoría deportiva, sólo se encontraron diferencias significativas entre las jugadoras y los jugadores del grupo G3 (Figura 2). 
Pradas de la Fuente, F; Carrasco Páez, L.; Martínez Pardo, E.; Herrero Pagán, R. (2007). Perfil antropométrico, somatotipo y composición corporal de jóvenes jugadores de tenis de mesa. Revista Internacional de Ciencias del Deporte. 7(3), 11-23 http://www.cafyd.com/REVISTA/00702.pdf

\begin{tabular}{|c|c|c|c|c|c|c|c|}
\hline GRUPOS & GÉNERO & ESTADÍSTICO & $x$ & $\mathrm{Y}$ & Endomorfia & Mesomorfia & Ectomorfia \\
\hline \multirow{6}{*}{$\begin{array}{c}\text { G1 } \\
\text { (benjamín) }\end{array}$} & \multirow{2}{*}{$\begin{array}{c}\text { MASCULINO } \\
n=15\end{array}$} & Media &,- 62 & 2,80 & 3,72 & 4,80 & 3,10 \\
\hline & & $\mathrm{Dt}$ & 2,63 & 2,28 & 1,43 & 87 & 1,36 \\
\hline & \multirow{2}{*}{$\begin{array}{c}\text { FEMENINO } \\
n=7\end{array}$} & Media &,- 88 & 1,04 & 4,17 & 4,25 & 3,29 \\
\hline & & Dt. & 3,10 & 2,39 & 1,63 & 1,13 & 1,54 \\
\hline & \multirow{2}{*}{$\begin{array}{l}\text { Total } \\
n=22\end{array}$} & Media &,- 70 & 2,24 & 3,86 & 4,63 & 3,16 \\
\hline & & Dt. & 2,72 & 2,41 & 1,47 & ,97 & 1,39 \\
\hline \multirow{6}{*}{$\begin{array}{c}\text { G2 } \\
\text { (alevín) }\end{array}$} & \multirow{2}{*}{$\begin{array}{c}\text { MASCULINO } \\
\quad \mathrm{N}=15\end{array}$} & Media &,- 45 & 2,19 ** & 3,80 & 4,67 & 3,35 \\
\hline & & Dt. & 2,27 & 1,48 & 1,37 & ,68 & ,98 \\
\hline & \multirow{2}{*}{$\begin{array}{l}\text { FEMENINO } \\
\mathrm{N}=12\end{array}$} & Media & $-1,91$ & 1,33 & 4,65 & 4,35 & 2,73 \\
\hline & & Dt. & 3,17 & 2,36 & 1,73 & 1,30 & 1,46 \\
\hline & \multirow{2}{*}{$\begin{array}{l}\text { Total } \\
N=27\end{array}$} & Media & $-1,10$ & 1,81 & 4,17 & 4,53 & 3,07 \\
\hline & & Dt. & 2,75 & 1,93 & 1,57 & ,99 & 1,23 \\
\hline \multirow{6}{*}{$\begin{array}{c}\text { G3 } \\
\text { (infantil) }\end{array}$} & \multirow{2}{*}{$\begin{array}{c}\text { MASCULINO } \\
N=8\end{array}$} & Media & ,37 & 1,90 & 3,12 & 4,26 & 3,50 \\
\hline & & Dt. & 2,63 & 1,42 & 1,51 & ,49 & 1,24 \\
\hline & \multirow{2}{*}{$\begin{array}{l}\text { FEMENINO } \\
\qquad N=6\end{array}$} & Media & $-1,33$ &,- 27 & 4,21 & 3,42 & 2,88 \\
\hline & & Dt. & 1,81 & 3,33 & ,76 & 1,20 & 1,23 \\
\hline & \multirow{2}{*}{$\begin{array}{l}\text { Total } \\
\mathrm{N}=14\end{array}$} & Media &,- 36 & ,97 & 3,59 & 3,90 & 3,23 \\
\hline & & Dt. & 2,40 & 2,57 & 1,33 & ,93 & 1,23 \\
\hline \multirow{6}{*}{ Total } & \multirow{2}{*}{$\begin{array}{l}\text { MASCULINO } \\
\qquad=38\end{array}$} & Media &,- 34 & $2,37 \#$ & 3,62 & 4,64 \#\# & 3,28 \\
\hline & & Dt. & 2,45 & 1,82 & 1,41 &, 74 & 1,18 \\
\hline & \multirow{2}{*}{$\begin{array}{l}\text { FEMENINO } \\
\qquad N=25\end{array}$} & Media & $-1,48$ & ,86 & 4,41 \#\# & 4,10 & 2,92 \\
\hline & & Dt. & 2,81 & 2,60 & 1,49 & 1,24 & 1,39 \\
\hline & \multirow{2}{*}{$\begin{array}{l}\text { Total } \\
N=63\end{array}$} & Media &,- 79 & 1,77 & 3,93 & 4,42 & 3,14 \\
\hline & & Dt. & 2,64 & 2,27 & 1,48 & ,99 & 1,27 \\
\hline
\end{tabular}

Tabla 2. Somatotipo y somatopuntos de los deportistas estudiados.

(**) Diferencias significativas para $\mathrm{p}<0,01$ al comparar entre grupos de género dentro de cada categoría; (\#) diferencias significativas para $\mathrm{p}<0,01$ al comparar entre géneros; (\#\#) diferencias significativas para $\mathrm{p}<0,05$ al comparar entre géneros.

El porcentaje muscular más elevado correspondió a la categoría infantil, existiendo diferencias significativas con respecto al grupo benjamín (Tabla 2). En cuando al análisis por sexos, solamente se observaron diferencias estadísticamente significativas entre varones y mujeres de la categoría infantil, con mayor magnitud en el sexo femenino (Figura 3). 
Pradas de la Fuente, F; Carrasco Páez, L.; Martínez Pardo, E.; Herrero Pagán, R. (2007). Perfil antropométrico, somatotipo y composición corporal de jóvenes jugadores de tenis de mesa. Revista Internacional de Ciencias del Deporte. 7(3), 11-23 http://www.cafyd.com/REVISTA/00702.pdf

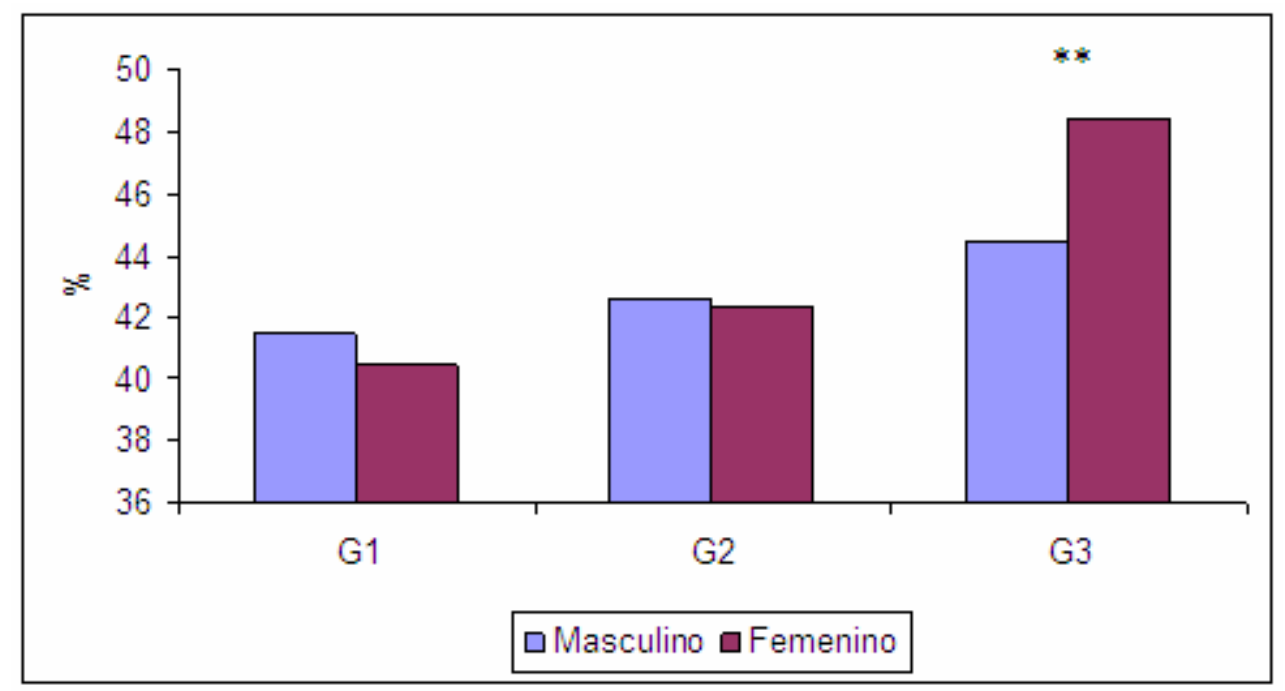

Figura 3. Componente muscular (porcentaje sobre la masa corporal) de los deportistas estudiados. ${ }^{* *} \mathrm{p}<0.01$.

El componente graso más alto, se encontró en el grupo benjamín, no existiendo diferencias relevantes entre categorías. Por otra parte, y atendiendo a una comparativa El análisis por grupos de género sí puso de manifiesto un porcentaje adiposo significativamente mayor en el sexo femenino respecto al grupo de varones $(16.60 \pm 5.40$ vs. $13.63 \pm 4.55 \%$, respectivamente; $\mathrm{p}<0.05)$. La evaluación de dicho componente corporal por sexos dentro de cada categoría deportiva, mostró mayores porcentajes de tejido adiposo subcutáneo en el grupo de jugadoras para todas las categorías, una diferencia muy próxima a significación estadística (Figura 4).

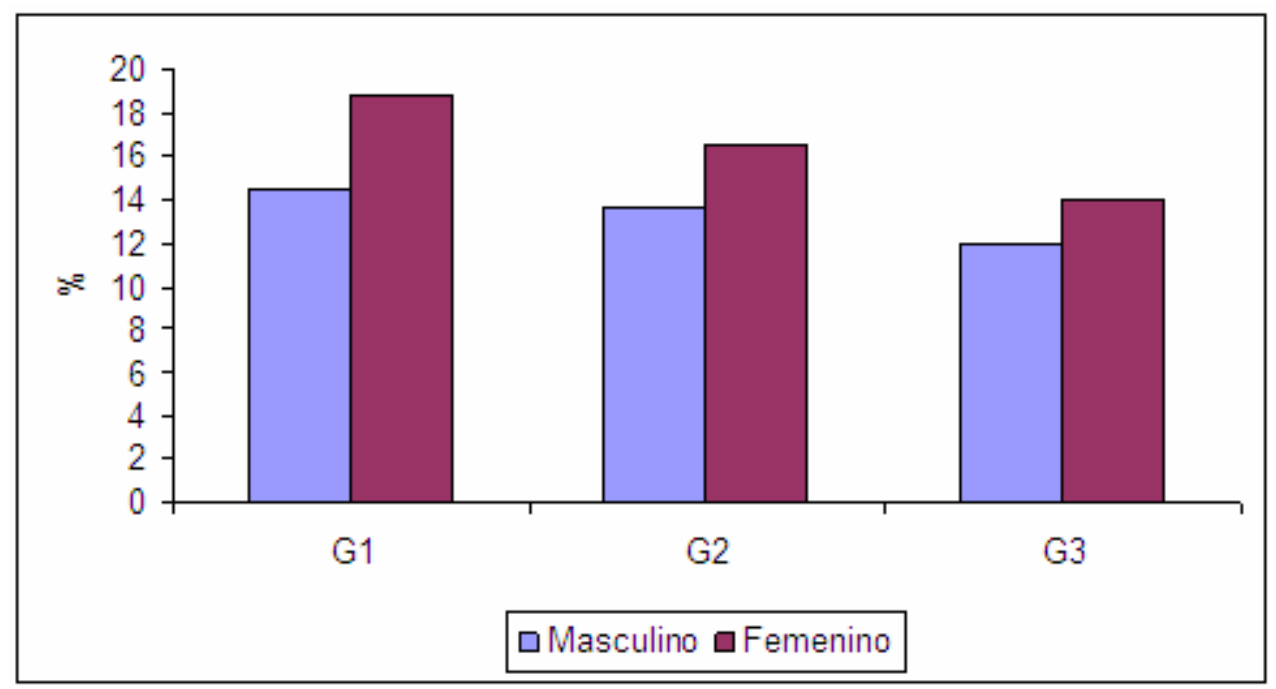

Figura 4. Componente graso (porcentaje sobre la masa corporal) de los deportistas estudiados. 
Pradas de la Fuente, F; Carrasco Páez, L.; Martínez Pardo, E.; Herrero Pagán, R. (2007). Perfil antropométrico, somatotipo y composición corporal de jóvenes jugadores de tenis de mesa. Revista Internacional de Ciencias del Deporte. 7(3), 11-23 http://www.cafyd.com/REVISTA/00702.pdf

En cuanto al componente residual, se observaron diferencias estadísticamente significativas al establecer la comparativa atendiendo, de forma exclusiva, a la variable género. En este sentido, se encontraron valores notablemente superiores en chicos que en chicas (23.56 \pm 1.19 vs. $21.61 \pm 3.35 \%$, respectivamente; $\mathrm{p}<0.01$ ). Estas diferencias quedaron también patentes al efectuar el análisis por sexos dentro de cada grupo (Figura 5).

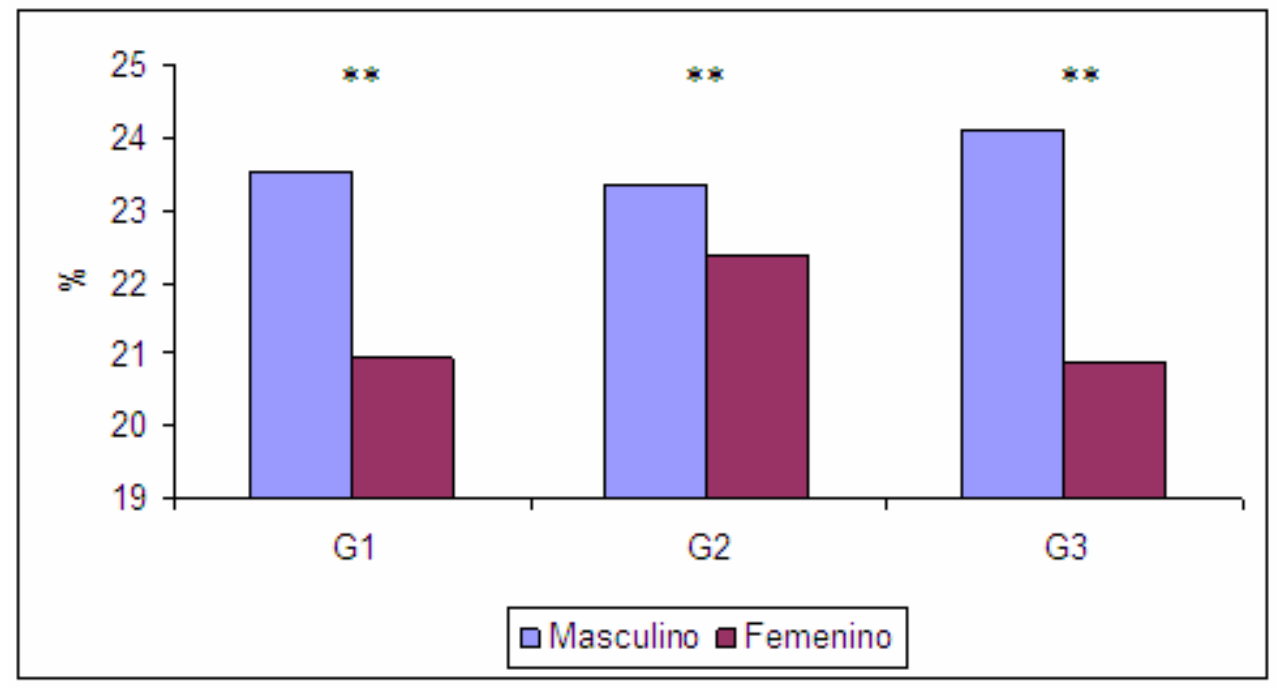

Figura 5. Componente residual (porcentaje sobre la masa corporal) de los deportistas estudiados.

El análisis del somatotipo de todo el grupo, mostró un predominio equilibrado de los componentes muscular y graso, aunque con ligera ventaja del compartimento muscular sobre el adiposo, tratándose, por tanto, de un somatotipo endomórfico-mesomórfico (Tabla 2 y Figura 6).

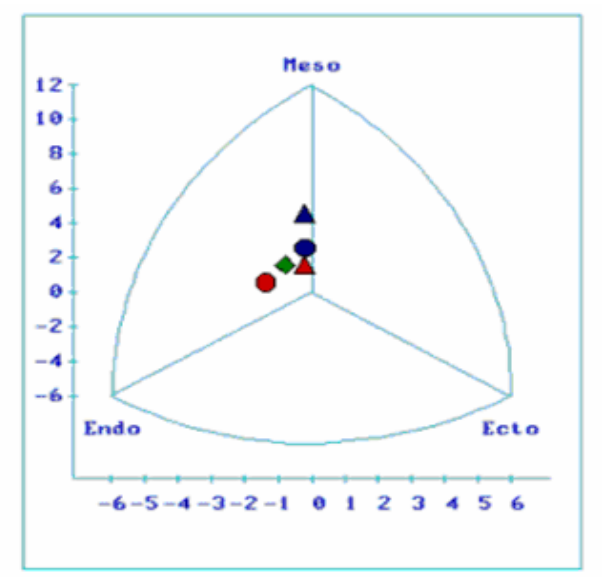

- Somatotipo de referencia (jugadores de tenis de mesa)

$\Delta$ Somatotipo de referencia (jugadoras de tenis de mesa)

Somatotipo global de los deportistas estudiados

Somatotipo de los jugadores de tenis de mesa estudiados.

Somatotipo de las jugadoras de tenis de mesa estudiadas.

Figura 6. Somatocarta del grupo estudiado y de un grupo de referencia (jugadores de tenis de mesa valorados en el Centro de Medicina Deportiva de Castilla y León). 
Pradas de la Fuente, F; Carrasco Páez, L.; Martínez Pardo, E.; Herrero Pagán, R. (2007). Perfil antropométrico, somatotipo y composición corporal de jóvenes jugadores de tenis de mesa. Revista Internacional de Ciencias del Deporte. 7(3), 11-23 http://www.cafyd.com/REVISTA/00702.pdf

El análisis del somatotipo de todo el grupo segmentado por sexos, mostró algunas diferencias con respecto a los resultados globales en los varones, que manifestaron un somatotipo mesomórfico balanceado.

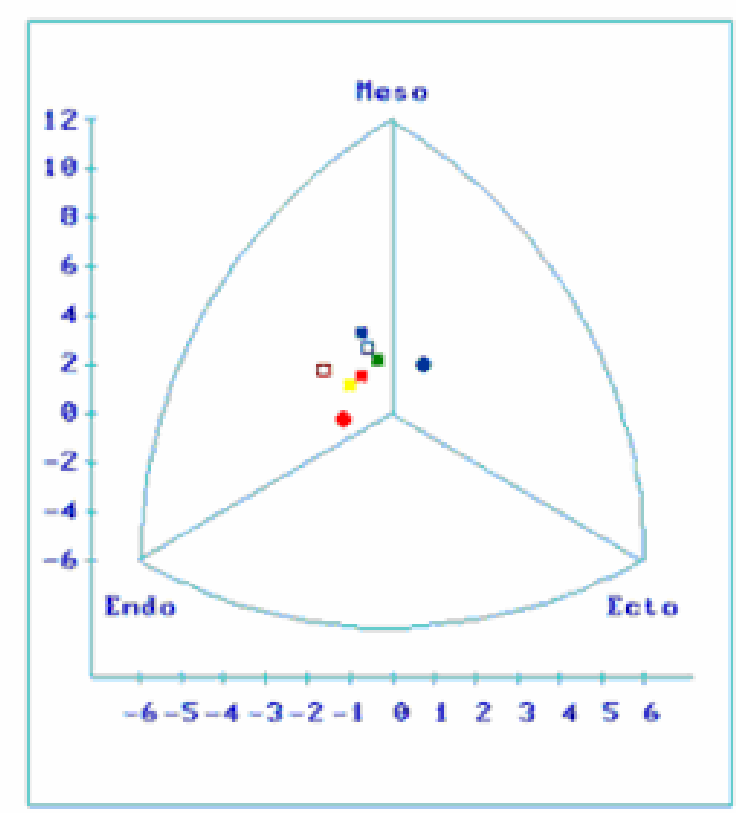

Somatotipo de refer encia (jugadores de ten is de mesa)

$\square$ Somatotipo de refer encia (jugadoras de ten is de mesa)

- Somatotipo de los jugadores perten ecientes al grupo G1.

- Somatotipo de las jug adoras pertenecientes al grupo G1.

- Somatotipo de los jugadores perten ecientes al grupo $\mathrm{G} 2$.

- Somatotipo de las jug adoras pertenecientes al grupo G2.

Somatotipo de los jugadores pertenecientes al grupo G3.

Somatotipo de las jug adoras pertenecientes al grupo $\mathrm{G} 3$.

Figura 7. Somatocarta de los deportistas estudiados distribuidos por grupos según su categoría y género.

La evaluación del somatotipo por categorías deportivas (Figura 7), no evidenció diferencias en relación a los valores de referencia, ya que la distancia de dispersión de dos somatotipos medios globales no superó en ningún caso los dos puntos (Tabla 3). A pesar de ello hay que destacar que el grupo infantil mostró un somatotipo mesomórfico balanceado, esto es, un predominio del compartimento muscular sobre los componentes grasos y de linealidad corporal, existiendo un equilibrio entre estos dos últimos. 
Pradas de la Fuente, F; Carrasco Páez, L.; Martínez Pardo, E.; Herrero Pagán, R. (2007). Perfil antropométrico, somatotipo y composición corporal de jóvenes jugadores de tenis de mesa. Revista Internacional de Ciencias del Deporte. 7(3), 11-23 http://www.cafyd.com/REVISTA/00702.pdf

\begin{tabular}{|c|c|c|c|}
\cline { 2 - 4 } \multicolumn{1}{c|}{} & \multicolumn{3}{c|}{ Grupos estudiados } \\
\hline Grupos de referencia & G1 & G2 & G3 \\
\hline Masculino & 0.24 & 0.14 & 0.71 \\
\hline Femenino & 0.43 & 0.73 & 0.73 \\
\hline
\end{tabular}

Tabla 3. Distancias de dispersión de dos somatotipos medios en los grupos estudiados considerando el factor género.

El análisis del somatotipo por subgrupos de género dentro de cada categoría deportiva, mostró en los varones de todas las categorías, un claro predominio del componente mesomórfico o muscular sobre los compartimentos endomórfico y ectomórfico. Por el contrario, el somatotipo del grupo femenino tanto de la categoría infantil, resultó ser meso-endomórfico, esto es, un predominio del compartimento graso sobre el muscular, y de ambos, sobre el componente de linealidad corporal; mientras que en las niñas benjamines y alevines se observó un somatotipo endomórifoco-mesomórfico, es decir, un predominio equilibrado de los componentes muscular y graso sobre el compartimento ectomórfico.

\section{Discusión}

Tal y como se esperaba, y de acuerdo con los datos aportados por los escasos trabajos realizados en jugadores de tenis de mesa, la determinación del somatotipo de los jugadores participantes en este estudio, ha mostrado un predominio claro del componente mesomórfico sobre los dos componentes restantes, lo cual ha resultado más notable en el grupo de varones.

Diversas investigaciones biotípicas realizadas en este deporte, han demostrado que los jugadores de tenis de mesa, suelen tener un buen desarrollo muscular, más evidente en las extremidades inferiores (muslos y piernas) (Matytsin, 1994).

En términos generales, desde el nacimiento hasta la adolescencia, la masa muscular aumenta de manera gradual, en relación a la ganancia de peso del sujeto. En el hombre, la masa muscular total aumenta desde el $25 \%$ del peso corporal hasta el 40 - $45 \%$ o más en la edad adulta. Una gran parte de esta ganancia se produce cuando el ritmo de desarrollo muscular llega a su máximo nivel en la pubertad, lo que se encuentra relacionado con la producción de testosterona, un hecho que concuerda con el mayor porcentaje muscular evidenciado en este estudio, en el grupo de categoría deportiva superior, es decir, en la categoría infantil, respecto a las otras dos categorías.

No obstante, el mayor porcentaje muscular en el subgrupo femenino de la categoría infantil, respecto al masculino del mismo grupo que ha podido observarse en el presente estudio, podría ser explicado por el reducido tamaño de los subgrupos comparados, unos resultados, que antes de ser extrapolados a la población de jugadores de tenis de mesa de la misma categoría deportiva, deberían ser contrastados ampliando el tamaño muestral. 
Pradas de la Fuente, F; Carrasco Páez, L.; Martínez Pardo, E.; Herrero Pagán, R. (2007). Perfil antropométrico, somatotipo y composición corporal de jóvenes jugadores de tenis de mesa. Revista Internacional de Ciencias del Deporte. 7(3), 11-23 http://www.cafyd.com/REVISTA/00702.pdf

Por otra parte, y atendiendo a la variable género, los resultados obtenidos en relación con la composición corporal mostraron un mayor porcentaje graso en mujeres respecto a varones, y un porcentaje óseo más alto en el grupo de varones con respecto al de mujeres, unos datos, que coinciden con otros estudios realizados en jugadores de tenis de mesa, en los que se pone de manifiesto el predominio equilibrado de los componentes muscular y graso sobre el componente ectomórfico (Astrand, 1992). En este sentido, hay que recordar que el componente graso o endomórfico es una variable muy importante en el rendimiento del jugador de tenis de mesa, ya que la grasa actúa como peso inerte en actividades donde la masa corporal debe ser desplazada de forma reiterada en contra de la gravedad, como ocurre durante los movimientos realizados en el transcurso de este juego. Además, la evidencia experimental en varios grupos etarios de diversas especialidades deportivas, ha demostrado una relación inversa entre masa grasa y rendimiento físico en actividades físicas que requieren el desplazamiento del peso del cuerpo tanto en dirección vertical (como en el salto), como horizontal (como la carrera). Es decir, el exceso de adiposidad corporal, suele influir negativamente en el rendimiento deportivo de este tipo de actividades, puesto que el aumento del peso corporal no se acompaña de un incremento paralelo de la capacidad para producir fuerza. Por tanto, teniendo en cuenta que la aceleración es directamente proporcional a la fuerza, pero inversamente proporcional a la masa corporal, el exceso de grasa a un nivel dado de aplicación de la fuerza, resultará en cambios más lentos en la velocidad y en la dirección, y este exceso de adiposidad también incrementa el costo metabólico de actividades físicas que implican el movimiento de la masa corporal (Shepard, 1998).

En definitiva, la mayoría de los deportes que implican la movilización de la masa corporal en contra de la gravedad, se benefician de un porcentaje graso relativamente bajo, tanto mecánica como metabólicamente, lo que concuerda con los resultados derivados de este trabajo, que han mostrado un compartimento endomórfico o graso, cuantitativamente menor que el muscular, tanto en el análisis global del grupo de estudio, como en los subgrupos de categorías deportivas.

Algunos resultados derivados de estudios de corte transversal indican que el porcentaje de grasa corporal se encuentra relacionado de forma inversa tanto con la capacidad aeróbica ( $\mathrm{VO}_{2}$ máx.) expresada relativamente al peso corporal, como con el rendimiento físico en carreras de larga distancia (Aziz, 2000). Éste mismo autor y sus colaboradores llevaron a cabo experimentos acerca de los efectos del incremento artificial del peso corporal sobre las respuestas fisiológicas al ejercicio, y sobre la capacidad de rendimiento físico, demostrando que la capacidad de carrera de individuos sanos, y de peso normal, se redujo cuando estos cargaban cinturones y chalecos con pesos.

No obstante, cantidades adecuadas de grasa, apropiadamente distribuidas, son ventajosas para algunos deportes como el rugby, y otros deportes de contacto en los cuales, la absorción de fuerza es importante.

En el caso del tenis de mesa, no se ha podido demostrar cuál es el porcentaje más apropiado de grasa para sus jugadores, entendiendo como apropiado, aquel que guarda una proporción ideal con el porcentaje magro corporal para la consecución de niveles óptimos de rendimiento físico-deportivo. 


\section{Conclusiones}

El componente mesomórfico es predominante sobre el resto de los componentes del somatotipo, un aspecto algo más evidente en los jugadores de género masculino.

A través del presente estudio, realizado con jóvenes jugadores nacionales, se ha podido demostrar un somatotipo global endomórfico-mesomórfico, coincidiendo con el de las categorías alevín e infantil. En cualquier caso, el somatotipo de los jugadores estudiados no muestra diferencias con el somatotipo de referencia empleado en este caso.

Los resultados de la composición corporal por grupos de género mostraron un mayor porcentaje graso en mujeres respecto a varones, y un porcentaje óseo más alto en el grupo de varones con respecto al de mujeres.

\section{Bibliografía}

Aziz A.R., Chia M. y Teh K.C. (2000). The relationship between maximal oxygen uptake and repeated sprint performance indices in field hockey and soccer players. J ournal of Sports Medicine and Physical Fitness, 40(3),195-200.

Astrand, P. O. y Rodahl, K. (1992). Fisiología del trabajo físico. Barcelona: Panamericana.

Bagur, C. y Serra, J.R. (1996). Clasificación de la actividad física y de los deportes mas comunes en función del trabajo que requieren. En: Serra, J.R. Prescripción de ejercicio físico para la salud. Barcelona: Paidotribo.

Bagur, C. (1996). Orientaciones básicas para programas de ejercicio físico de ámbito no competitivo. En: Serra, J.R. Prescripción de ejercicio físico para la salud. Barcelona: Paidotribo,

Bermejo, J.L., Quintano, J., Ramos, M., Don, Z. (1991). Tenis de mesa. Madrid: Comité Olímpico Español.

Bompa, T. (1987). La selección de atletas con talento. Revista de Entrenamiento Deportivo, 1(2), 46-54.

Carter, J.E.L. y Heath B.H. (1990). Somatotyping. Development and applications. Cambridge: Cambridge University Press.

De Rose, E.H. y Guimaraes, A.C. (1980). A model for optimization of somatotype in young athletes. En: Ostin, M., Buenen, G., Simons, J.: Kinanthropometry II. Baltimore: University Park Press.

Esparza, F. (1993). Manual de cineantropometría. Pamplona: GREC-FEMEDE.

Matytsin, O.V. (1994). The role of personal characteristics of the table tennis player in providing efficiency and stability during competitions. International Journal of Table Tennis Sciences, 2, 55-60.

Maximiliano, G. (2001). Componentes del somatotipo y ecuaciones antropométricas. Apunts, 137, 5-15. 
Pradas de la Fuente, F; Carrasco Páez, L.; Martínez Pardo, E.; Herrero Pagán, R. (2007). Perfil antropométrico, somatotipo y composición corporal de jóvenes jugadores de tenis de mesa. Revista Internacional de Ciencias del Deporte. 7(3), 11-23 http://www.cafyd.com/REVISTA/00702.pdf

Mazza, J.C. (1993). Mediciones antropométricas. Estandarización de las técnicas de medición, actualizada según parámetros internacionales. Revista de Actualización en Ciencias del Deporte, 1(2).

Ross, W.D. y Marfell-Jones, M.J. (1982). Kinanthropometry. En : Mac Dougall, J.D., Winger, H.A. y Green, M.J.: Physiological Testing of Elite Athlete. New York: Mouvement Publications Inc.

Shephard, K. y Astrand, P. (1998). La resistencia en el deporte. Barcelona: Paidotribo.

Zatsiorski, V. (1989). Metrología deportiva. Moscú: Planeta. Moscú. 